\section{A) Check for updates}

Cite this: Nanoscale, 2020, 12, 18455

\title{
Fractionation of block copolymers for pore size control and reduced dispersity in mesoporous inorganic thin films $\uparrow$
}

\author{
Alberto Alvarez-Fernandez, (D) a Barry Reid, (D) a Jugal Suthar, (D) a,b Swan Yia Choy, ${ }^{a}$ \\ Maximiliano Jara Fornerod, (ID a Niamh Mac Fhionnlaoich, (D) a Lixu Yang, (ID) a \\ Benjamin Schmidt-Hansberg (iD c and Stefan Guldin iD *a
}

\begin{abstract}
Mesoporous inorganic thin films are promising materials architectures for a variety of applications, including sensing, catalysis, protective coatings, energy generation and storage. In many cases, precise control over a bicontinuous porous network on the $10 \mathrm{~nm}$ length scale is crucial for their operation. A particularly promising route for structure formation utilizes block copolymer (BCP) micelles in solution as sacrificial structure-directing agents for the co-assembly of inorganic precursors. This method offers pore size control via the molecular weight of the pore forming block and is compatible with a broad materials library. On the other hand, the molecular weight dependence impedes continuous pore tuning and the intrinsic polymer dispersity presents challenges to the pore size homogeneity. To this end, we demonstrate how chromatographic fractionation of BCPs provides a powerful method to control the pore size and dispersity of the resulting mesoporous thin films. We apply a semi-preparative size exclusion chromatographic fractionation to a polydisperse poly(isobutylene)-block-poly(ethylene oxide) (PIB- $b$ - PEO) BCP obtained from scaled-up synthesis. The isolation of BCP fractions with distinct molecular weight and narrowed dispersity allowed us to not only tune the characteristic pore size from $9.1 \pm 1.5$ to $14.1 \pm 2.1 \mathrm{~nm}$ with the identical BCP source material, but also significantly reduce the pore size dispersity compared to the non-fractionated BCP. Our findings offer a route to obtain a library of monodisperse BCPs from a polydisperse feedstock and provide important insights on the direct relationship between macromolecular characteristics and the resulting structure-directed mesopores, in particular related to dispersity.
\end{abstract}

\author{
Received 9th July 2020, \\ Accepted 21st August 2020 \\ DOI: $10.1039 / d 0 n r 05132 b$ \\ rsc.li/nanoscale
}

\section{Introduction}

Structural control over a bicontinuous porous inorganic network on the $10 \mathrm{~nm}$ length scale is crucial for the functioning of many mesoporous thin film architectures, as demonstrated for mesoporous sensors, ${ }^{1-3}$ (photo)catalysis, ${ }^{4,5}$ photovoltaics, including perovskite ${ }^{6}$ and dye-sensitized solar cells, ${ }^{7}$ optical coatings, ${ }^{8}$ protective barriers ${ }^{9}$ and separation and purification membranes. ${ }^{10,11}$ Besides the mean pore size, the resulting pore dispersity is another important material property, as evidenced in examples related to electrochemistry, ${ }^{2}$ sensing, ${ }^{12,13}$ and controlled release. ${ }^{14,15}$ This is best showcased

\footnotetext{
${ }^{a}$ Department of Chemical Engineering, University College London, Torrington Place, London,WC1E 7JE, UK. E-mail: s.guldin@ucl.ac.uk

${ }^{b}$ UCL School of Pharmacy, University College London, 29-39 Brunswick Square, Bloomsbury, London, WC1N 1AX, UK

${ }^{c}$ BASF SE, Process Research \& Chemical Engineering, Coating \& Film Processing, Carl-Bosch-Strasse 38, 67056 Ludwigshafen am Rhein, Germany

$\dagger$ Electronic supplementary information (ESI) available: Additional structural analysis results using GISAXS, AFM and EP. See DOI: 10.1039/d0nr05132b
}

in mass transport-limited scenarios governed by the Knudsen diffusion. ${ }^{16,17}$

To this end, block copolymer (BCP) based co-assembly represents a promising bottom-up approach to create ordered mesoporous structures with tunable size and morphology ${ }^{18-21}$ In the so-called persistent micelle templating method, inorganic precursors (typically sol-gel derived) are embedded into the corona of BCP micelles via preferential supramolecular interactions. ${ }^{22}$ In a subsequent step, the hybrid composite can be transformed into an ordered inverse opal-type mesoporous structure by either thermal calcination, ${ }^{22}$ or various physical or chemical treatments including UV-ozone degradation, ${ }^{23}$ oxygen etching ${ }^{24}$ or photocatalytic reactions. $^{25}$

The use of BCP as structure-directing agent offers reliable control over porosity and pore size. While porosity is commonly tuned by the mixing ratio between organic and inorganic precursor, the pore size is predominantly determined by the molecular weight of the sacrificial block forming the micelle core,${ }^{26,27}$ leading to final pore dimensions in the range of 5 to $50 \mathrm{~nm}$. 
Several recent studies have identified the close relationship between the BCP dispersity and the size distribution of the obtained co-assembled features ${ }^{28-30}$ highlighting the requirement of precise control over the macromolecular characteristics of the structure-directing agent. In general, the synthesis of BCPs with bespoke molecular weight and narrow dispersity $(\nexists)$ is an elaborate process that involves multiple steps, some of which are furthermore difficult to govern. Synthesis is either conducted by sequential addition of monomers via living/controlled chain-growth polymerization procedures ${ }^{30,31}$ or relies on coupling reactions between different end-functionalized chains segments. For the former, close control must be achieved over reaction dynamics and conversion efficiency in order to obtain defined molecular weights and low polydispersity. ${ }^{32}$ Anionic and cationic polymerization ${ }^{33}$ as well as some types of controlled radical polymerization ${ }^{34}$ have been successfully employed in the synthesis of low polydisperse BCPs. The second strategy requires end-group functionalization for effective coupling, e.g. via Diels-Alders, thiol-ene or cycloaddition reactions. ${ }^{35}$ These coupling reaction must be rapid and selective to ensure efficient linking between the chains. ${ }^{36}$ In all cases, synthesis is highly sensitive to impurities and precise reactions conditions. Furthermore, adequate purification processes are necessary for the employed reagents, making the synthesis procedure overall very sensitive and time consuming. ${ }^{32}$

Size exclusion chromatographic (SEC) fractionation is routinely applied for purifying BCPs from their side reaction products, mainly unreacted precursors or unlinked homopolymers. ${ }^{37-40}$ More recently, Park et al. have extended liquid chromatography to the separation of BCPs in terms of their molecular weights. ${ }^{41}$ Fractions containing polystyreneblock-polyisoprene copolymers with narrower distribution in molecular weight and chemical composition were collected using a HPLC with two separation stages, showing the great potential of this approach to obtain an extensive BCP library with reduced dispersity and synthetic effort. Further experiments have established chromatography fractionation as a reliable and effective technique to provide systematic control over the macromolecular parameters (i.e. molecular weight and polydispersity) of the BCP. ${ }^{28,29,42,43}$

In this work, we present the application of BCP chromatographic fractionation to create tailored inorganic mesoporous thin films. Crucially, we identify the role of molecular weight, composition and dispersity on the resulting pore size distribution. We first isolate different BCP fractions from a polydisperse amphiphilic poly(isobutylene)-block-poly(ethyleneoxide) (PIB- $b$-PEO) sample and characterize their molecular weight and composition via gel permeation chromatography (GPC) and nuclear magnetic resonance (NMR). Different BCP fractions then serve for formulation with aluminosilicate sol. After deposition, the obtained thin films are characterized in both hybrid and calcined form via grazing incidence small angle X-ray scattering (GISAXS). Atomic force microscopy (AFM) and ellipsometric porosimetry (EP) to enable a full validation of this approach.

\section{Experimental}

\section{Materials}

$\mathrm{PIB}_{39}$-b-PEO36 BCP $\left(M_{\mathrm{n}} 4.85 \mathrm{~kg} \mathrm{~mol}^{-1}\right.$; polydispersity index 1.26) was supplied by BASF following a previously reported synthetic route. ${ }^{44}$ Size exclusion chromatography (SEC) was chosen as the isolation technique for the different molecular weight $\left(M_{\mathrm{w}}\right)$ BCPs and was conducted with an Akta Explorer instrument (GE Life Sciences, Sweden). A semipreparative column (Tricorn 10/300, GE Life Sciences, Sweden) packed with Toyopearl HW-50F (Tosoh, Japan) served as the stationary phase and methanol was used as mobile phase. The sample concentration was set to $200 \mathrm{mg} \mathrm{mL}^{-1}$ and the flowrate of the mobile phase at $1 \mathrm{~mL} \min ^{-1}$. Samples were injected manually using an INV-907 injection valve equipped with a $500 \mu \mathrm{l}$ sample loop. The chromatograms were recorded with a UV-900 UV absorption detector operating at a wavelength of $280 \mathrm{~nm}$. Fractions of $1 \mathrm{ml}$ in volume were collected with a fraction collector (Frac-950, GE Life Sciences, Sweden). The solvent was removed from the fractions by evaporation.

\section{BCP characterization}

${ }^{1} \mathrm{H}-\mathrm{NMR}$ spectra were recorded on either a Bruker Advance III (600 MHz) or Bruker Advance Neo (700 MHz) NMR spectrometer at room temperature with $\mathrm{CDCl}_{3}$ as solvent. GPC was conducted at room temperature, using $5 \mu \mathrm{m}$ PSS SDV medium combination columns $(300 \mathrm{~mm} \times 9 \mathrm{~mm})$ with $\mathrm{THF}$ as solvent. Measurements were performed at a flow rate of $1 \mathrm{~mL} \mathrm{~min}{ }^{-1}$ with a differential refractometer RI and a multi-angle light scattering detector (Brookhaven Instruments Bi-MwA). The elution times were converted into molecular weights via a calibration curve based on low dispersity polystyrene standards.

\section{Preparation of mesoporous aluminosilicate films}

Inorganic sol material and mesoporous inorganic aluminosilicate films were prepared as described elsewhere. ${ }^{8,25}$ BCP samples were first dissolved in a toluene/1-butanol azeotrope solution before mixing with the inorganic sol in an organic/inorganic ratio of $2: 8$. All samples were spin-coated at $2000 \mathrm{rpm}$ for 20 seconds and immediately annealed on a programmable hot plate using a ramp rate of $1^{\circ} \mathrm{C}$ per min to a final temperature of $130{ }^{\circ} \mathrm{C}$ for 30 minutes. To remove the organic structuredirecting agent, samples were calcined in a muffle furnace at $450{ }^{\circ} \mathrm{C}$ for 1 hour.

\section{Characterization of mesoporous aluminosilicate films}

A Semilab SE2000 spectroscopic ellipsometer was used to perform spectroscopic ellipsometry (SE) and ellipsometric porosimetry (EP) measurements. All SE and EP data analysis was carried out on the Semilabs SEA software (v1.6.2). Prior to EP measurements, samples were placed on a hotplate at $120{ }^{\circ} \mathrm{C}$ for 10 minutes. This was to ensure that no residual atmospheric water molecules remained in the pores prior to measurement. EP porosity isotherms were derived from the evolution of the refractive index value as a function of relative humidity in the sample chamber. ${ }^{45}$ Application of the Lorentz- 
Lorentz effective medium approximation allowed accurate determination of the overall porosity of each sample based on the change in refractive index as a consequence of capillary condensation inside the mesopores. ${ }^{46}$ The incremental onset of capillary condensation was related to the pore diameter of the material via the Kelvin Equation. ${ }^{45,47}$ All pore size and pore volume measurements were derived from the adsorption branches of the EP porosity isotherm and adjusted based on the assumption of an ellipsoidal pore shape due to film contraction during template removal. ${ }^{45}$

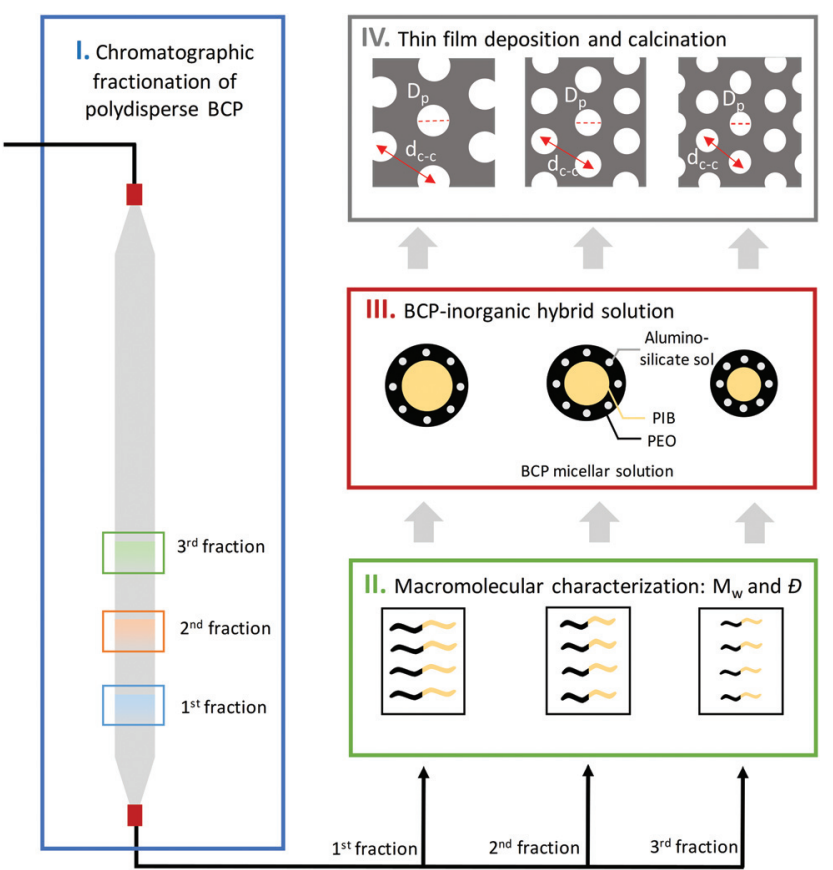

Fig. 1 Schematic illustration of the BCP fractionation process. A polydisperse $B C P$ is split into several molecular weight fractions by size exclusion chromatography. In a subsequent step, the BCP fractions serve as structure-directing agent to create mesoporous materials with tuneable pore sizes.
GISAXS data were acquired on a SAXSLab Ganesha wit at an incidence angle of 0.2. GISAXS data analysis was performed using FitGISAXS. Atomic Force Microscopy (AFM) images were obtained from a Bruker Dimension Icon atomic force microscope with a Bruker ScanAsyst Air probe (nominal tip radius $2 \mathrm{~nm}$ ) in ScanAsyst mode.

\section{Results and discussion}

Several molecular weight $\left(M_{\mathrm{w}}\right)$ BCP were isolated following the methodology sketched in Fig. 1. In a first step, a concentrated solution of the polydisperse BCP was injected into the system. Steric and chemical interactions between the hydroxylated methacrylate-based column and the polydisperse BCP resulted in a fractionation of the feedstock. These then served as sacrificial host for the co-assembly of inorganic guest material. $0.5 \mathrm{ml}$ of a $200 \mathrm{mg} \mathrm{ml} \mathrm{m}^{-1}$ solution of the PIB- $b$-PEO BCP in $\mathrm{MeOH}$ was first injected into the SEC system and subsequently

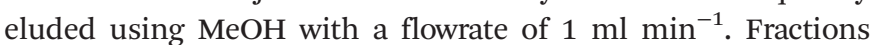
were taken automatically every minute. Fig. 2A displays the SEC chromatogram of the polydisperse PIB- $b$-PEO recorded by a UV detector. The elution was divided into twenty-three fractions as shown in Fig. 2A by segmenting the retention volume every $1 \mathrm{ml}$. The BCP appeared between retention volume $V_{\mathrm{R}}=16$ to $21 \mathrm{ml}$. Only one broad peak was observed. The obtained fractions were heated at $65^{\circ} \mathrm{C}$ overnight in order to evaporate the $\mathrm{MeOH}$. Note that the fractions A16 and A20 at the respective tails of the elution peak did generally not provide sufficient material to perform the macromolecular characterization, hence only fractions A17, A18 and A19 were considered for further experiments. In order to determine the composition and molecular weight of the isolated fractions ${ }^{1} \mathrm{H}$ NMR and GPC experiments were carried out. Fig. 2B shows the GPC traces of the assynthesized PIB- $b$-PEO BCP (black), and the three fractions studied during this work A17 (blue), A18 (green) and A19 (red).

Table 1 lists all the important macromolecular characteristics of the samples studied during this work. The molecular
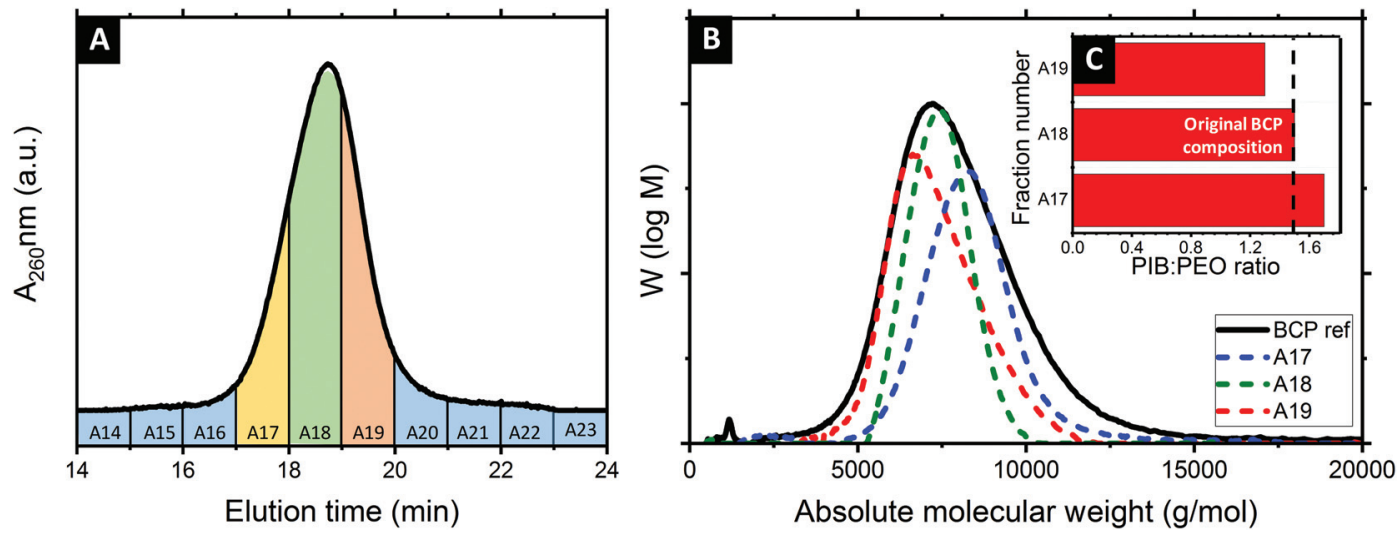

Fig. 2 (A) Isothermal SEC profile of the as-synthesized BCP. (B) Overlay of the GPC chromatograms of the non-fractionated PIB- $b$-PEO block copolymer starting material (black line) and the three fractions isolated during the fractionation process, A17 (blue); A18 (green) and A19 (red). (C) PIB : PEO composition of each fraction from ${ }^{1} \mathrm{H}$ NMR. 
Table 1 Macromolecular characteristics of the non-fractionated BCP used as starting material and the different fractions isolated during the process

\begin{tabular}{lllll}
\hline Fraction & A17 & A18 & A19 & BCP $_{\text {ref }}$ \\
\hline$M_{\mathrm{W}}\left(\mathrm{kg} \mathrm{mol}^{-1}\right)^{a}$ & 8.3 & 7.4 & 6.6 & 7.2 \\
$M_{\mathrm{W}}\left(\mathrm{kg} \mathrm{mol}^{-1}\right)^{b}$ & 8.1 & 7.5 & 6.7 & 7.7 \\
$M_{\mathrm{W}}$ PIB $\left(\mathrm{kg} \mathrm{mol}^{-1}\right)^{b}$ & 5.5 & 5.0 & 4.2 & 5.0 \\
$M_{\mathrm{W} \text { PEO }\left(\mathrm{kg} \mathrm{mol}^{-1}\right)^{b}}$ & 2.6 & 2.5 & 2.5 & 2.7 \\
$D$ & 1.10 & 1.12 & 1.11 & 1.25
\end{tabular}

${ }^{a}$ Calculated by GPC. ${ }^{b}$ Determined by NMR taking the signal of the phenyl linkage as reference.

weight $\left(M_{\mathrm{w}}\right)$ of the non-fractionated BCP was determined to be $7.2 \mathrm{~kg} \mathrm{~mol}{ }^{-1}$ (via a calibration curve based on low dispersity polystyrene standards), with a dispersity $Ð=1.25$. In comparison, the three fractions exhibited a $M_{\mathrm{w}}$ of $8.3 \mathrm{~kg} \mathrm{~mol}^{-1}$ (A17), $7.4 \mathrm{~kg} \mathrm{~mol}{ }^{-1}$ (A18) and $6.6 \mathrm{~kg} \mathrm{~mol}{ }^{-1}$ (A19), respectively. It is important to highlight that $Ð \approx 1.1$ was calculated for each of the collected fractions, a significantly narrower distribution than the as-synthesized $\mathrm{BCP}(\nexists \approx 1.25)$.

The degree of polymerization of the two blocks (PIB and PEO), obtained by ${ }^{1} \mathrm{H}$ NMR with taking the signal of the phenyl linkage as reference (see Fig. S1 and Table S1, ESI $\dagger$ ), was found to vary between the several collected fractions. The subsequently calculated PIB : PEO molar ratio corresponds to 1.7 (A17), 1.5 (A18) and 1.3 (A19), respectively. This compares to a molar ratio of 1.5 determined for the as-synthesized BCP. One may argue whether the increasing relative amount of PEO may be related to preferential interactions via hydrogen bonding between the PEO block and the hydroxylated methacrylate column packing material. However, when calculating the absolute molecular weight, $M_{\mathrm{w}}$ of the PEO block remained somewhat constant between the fractions, while the $M_{\mathrm{w}}$ of the PIB varied significantly. It is therefore reasonable to assume that the observed polydispersity is related to the PIB phenol precursor material rather than the subsequent grafting of polyethylene oxide. ${ }^{8,48}$ Four BCPs served as structure-directing agents during this work. The three monodisperse BCP fractions (A17, A18, A19) were prepared alongside the polydisperse, non-fractionated BCP for comparison. To this end, BCP samples were first dissolved in a toluene/1-butanol azeotrope solution before mixing with the inorganic sol. In a subsequent step, samples were spin-coated at $2000 \mathrm{rpm}$ for $30 \mathrm{~s}$. The hybrid aluminosilicate-BCP thin films obtained were first studied by 2D GISAXS (see ESI, Fig. S2 $\uparrow$ for diffraction patterns and line-cuts along $q_{\mathrm{y}}$ integrated around the Yoneda band). The diffraction ring found in all cases is consistent with the presence of poly-oriented domains. The position of the first Bragg peak $\left(q^{*}\right)$ enabled to calculate the centre-to-centre pore distance $\left(d_{\mathrm{c}-\mathrm{c}}\right)$, with $29.3 \mathrm{~nm}$, $25.2 \mathrm{~nm}$ and $23.8 \mathrm{~nm}$ obtained for samples co-assembled by A17, A18 and A19, respectively. Hence, the decreasing $M_{\mathrm{w}}$ of the pore forming block directly manifested in a reduced feature size in the hybrid film.

Fig. 3A-D shows the topographical AFM images of the mesoporous aluminosilicate films after BCP removal in a calcination step. The centre-to-centre distance $\left(d_{\mathrm{c}-\mathrm{c}}\right)$ was calculated
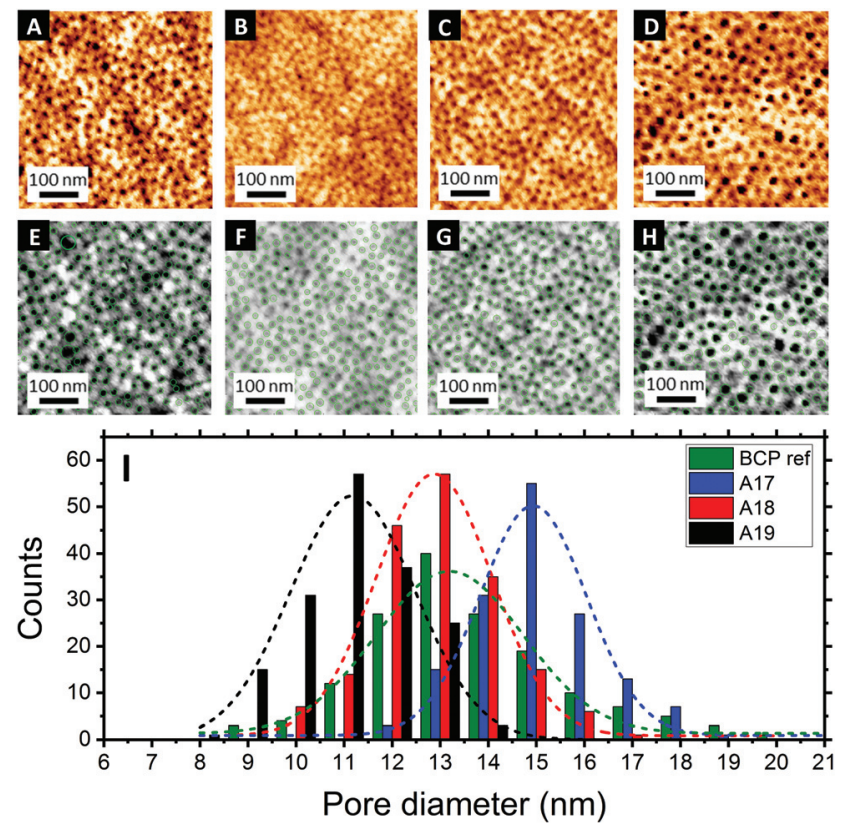

Fig. 3 AFM topographical images of the mesoporous films obtained using the non-fractionated $\mathrm{BCP}(\mathrm{A})$ and the three different $\mathrm{BCP}$ fractions isolated during the fractionation process A19 (B), A18 (C) and A17 (D). $(\mathrm{E}-\mathrm{H})$ Image overlay with pore recognition.(I) Corresponding pore diameter histograms obtained by image analysis.

for each sample using the correlation peak of the power spectral density (PSD) (see Fig. S3, ESI $\dagger$ ). Again, a clear relationship between the $M_{\mathrm{w}}$ of the BCP and the resulting $d_{\mathrm{c}-\mathrm{c}}$ was found, with $d_{\mathrm{c}-\mathrm{c}}=26.1 \mathrm{~nm}$ for the non-fractionated $\mathrm{BCP}$ and $d_{\mathrm{c}-\mathrm{c}}=$ $31.9 \mathrm{~nm}, 26.6 \mathrm{~nm}$ and $23.8 \mathrm{~nm}$ for the three fractions A17, A18 and A19, respectively.

Image analysis of the topographic AFM images was carried out using the software Pebbles ${ }^{49}$ to provide quantitative information about the in-plane pore dimension $\left(D_{\mathrm{i}-\mathrm{p}}\right)$ on the sample surface. The obtained pore size distribution histograms showed a constant increase with the molecular weight of the $D_{\mathrm{i}-\mathrm{p}}$ for the different BCP isolated fractions, i.e. $10.9 \pm$ $1.4 \mathrm{~nm}$ for A19, $12.3 \pm 1.5 \mathrm{~nm}$ for A18 and $14.8 \pm 1.6 \mathrm{~nm}$ for A17, and with significantly reduced pore dispersity when compared to the non-fractionated BCP $(13.0 \pm 3.6)$. Sample analysis by AFM is limited to the surface topography and, thus, alternative characterization techniques are required to evaluate the obtained results. ${ }^{50}$

To this end, ellipsometric porosimetry (EP) has emerged as a reliable characterization technique to probe porosity, pore size and pore morphology of thin films. ${ }^{50}$ Fig. 4 (left column) shows the EP adsorption isotherms obtained for the respective samples. Please note that the films exhibited a porosity in the range of $47 \%$ to $54 \%$. Analysis of the adsorption isotherms allowed to determine the pore size distribution of the mesoporous thin films via a modified Kelvin equation. ${ }^{45}$ The Isotropic Inorganic Pore Contraction model ${ }^{45}$ was applied to calculate the in-plane $\left(D_{\mathrm{i}-\mathrm{p}}\right)$ and out-of-plane $\left(D_{\mathrm{o}-\mathrm{p}}\right)$ pore diameter distribution (PDD) data based on the EP measurements. 

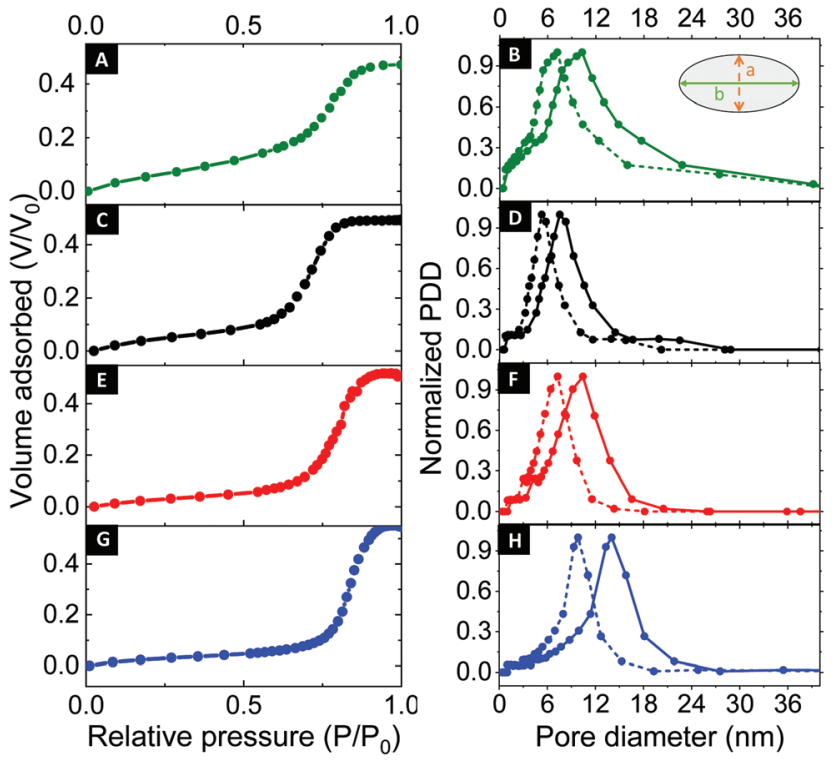

Fig. 4 EP adsorption isotherms for the as-synthesized BCP and the different fractions with correlated pore size distributions: non-fractionated $B C P(A, B), A 19(C, D), A 18(E, F)$ and $A 17(G, H)$.

Fig. 4 (right column) shows the PDD for the mesoporous aluminosilicate samples created using different $M_{\mathrm{w}}$ BCP. In line with AFM observations, a notable increase in $D_{\mathrm{i}-\mathrm{p}}$ was found with larger BCP molecular weight. With the lower $M_{\mathrm{w}}$ BCP (A19) exhibited a $D_{\mathrm{i}-\mathrm{p}}$ of $9.1 \pm 1.5 \mathrm{~nm}$, the medium BCP (A18) displayed a $D_{\mathrm{i}-\mathrm{p}}$ of $11.9 \pm 1.9 \mathrm{~nm}$, and the largest BCP (A17) resulted in a $D_{\mathrm{i}-\mathrm{p}}$ of $14.1 \pm 2.1 \mathrm{~nm}$. The $D_{\text {o-p }}$ dimensions scaled accordingly with $4.7 \pm 0.9 \mathrm{~nm}$ (A19); $8.3 \pm 1.1 \mathrm{~nm}$ (A18) and 9.2 $\pm 1.3 \mathrm{~nm}$ (A17). These results provide a clear indication of the correlation between the pore size and the $M_{\mathrm{w}}$ of the BCP used as structure-directing agent. In order to study the dependence between both parameters, the results were fitted to the following scaling law: $R_{\mathrm{p}}=C N_{\mathrm{PEO}}^{\alpha} N_{\mathrm{PIB}}^{\beta}$, where $R$ is the pore radius, $C$ is a constant, and $N$ represents the degree of polymerization of each block. ${ }^{51}$ Since $N_{\text {PEO }}$ was found approximately constant for the three different fractions, the relation can be simplified as $R_{\mathrm{p}}$ $=C^{\prime}\left[\mathrm{N}_{\mathrm{PIB}}^{\beta}\right]$. Indeed, a linear variation with a $\beta=0.8$ was previously found for excluded-volume BCP micelles, ${ }^{52,53}$ confirming the above-mentioned scaling law (see Fig. 5A). An analogous dependence of the resulting pore size with the molecular weight of one of the BCP blocks was recently observed in $\mathrm{TiO}_{2}$ mesoporous films made from a library of poly $(N, N$-dimethylacrylamide)- $b$-polystyrene BCP synthesized via reversible addition-fragmentation chain transfer polymerization. ${ }^{54}$ However, while previous approaches required extensive synthetic efforts to obtain a BCP library, the herein presented chromatographic fractionation enables systematic studies on structure-function relationships from a single polydisperse feedstock with little preparative work (see ESI, Fig. S4 †).

Moreover, analysis of EP desorption isotherms provided information about the size of the pore necks, i.e. the narrow restrictions that are connecting pores within the network. ${ }^{45,55,56}$ As shown in the ESI (Fig. S5 †), the mesoporous films prepared using the lowest $M_{\mathrm{w}} \mathrm{BCP}$ as structure-directing agent (A19) exhibited pore necks of $\approx 4.5 \mathrm{~nm}$, which expanded to $\approx 5.8 \mathrm{~nm}$ and $\approx 7.3 \mathrm{~nm}$ for the medium (A18) and larger (A17) sized structure-directing agent, respectively. To summarize, all structural parameters obtained by the different techniques are listed in Table 2.

Notably, when comparing the fractionated to the non-fractionated results, a clear narrowing of the pore size distribution is apparent. For AFM, the dispersity $D$ of the surface-accessible mesopores, defined as (coefficient of variance) ${ }^{2}+1,{ }^{57}$ was found to be 1.08 for the non-fractionated case, which reduced as a consequence of fractionation to 1.02 (A19), 1.01 (A18) and 1.01 (A17), respectively. Alongside, EP of the non-fractionated BCP (Fig. 4A and B) provided a less defined adsorption isotherm, indicating a more polydisperse porous structure. Indeed, when comparing the results obtained for the non-fractionated BCP $\left(D_{\mathrm{i}-\mathrm{p}}\right.$ of $10.7 \pm 4.8 \mathrm{~nm}$ and $D_{\text {o-p }}$ of $\left.7.7 \pm 3.7 \mathrm{~nm}\right)$ with the fractionated samples, a clear improvement in the pore size dispersity was observed. In this case, the pore size dispersity $D$, reduced from 1.20 to 1.03 (A19), 1.03 (A18) and 1.02 (A17) as a consequence of fractionation (see Fig. 5B).
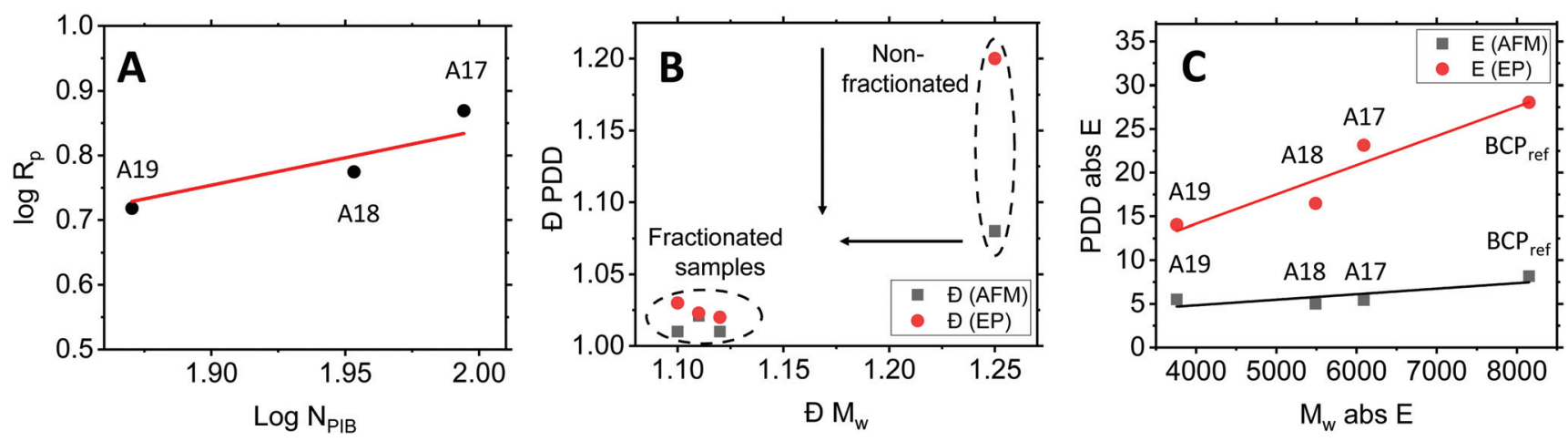

Fig. 5 (A) Logarithmic variation of the pore radius as a function of NPIB for the three different fractions studied in this work. (B) Correlation between BCP $M_{w}$ and pore size dispersity $\Theta$ for the different polymers studied in this work. (C) Correlation between BCP $M_{w}$ and pore size entropy for the different polymers studied in this work. 
Table 2 Structural parameters (pore center-to-center distance $\left(d_{c-c}\right)$, in-plane pore diameter $\left(D_{\text {i-p }}\right)$, out-of-plane pore diameter $\left(D_{\mathrm{o}-\mathrm{p}}\right)$, porosity and pore neck diameter (PND)) determined by the different techniques us ed during this study, i.e. AFM, GISAXS and EP

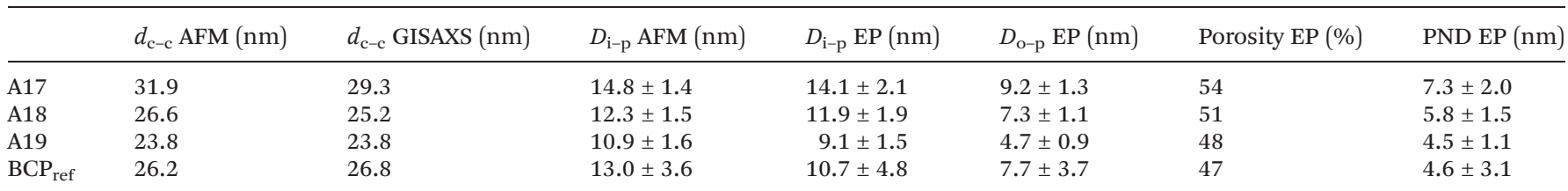

While reporting of $Ð$ is common practice in the community, it requires the population to follow a prescribed distribution. This may not be the case for mesopores, where poly- or heterodisperse pore sizes are often found. ${ }^{9,58}$ Indeed, the pore sizes obtained by EP herein could not be precisely described by normal distributions (see ESI, Fig. S6†). We have recently introduced the concept of information entropy to report on nanoparticle dispersity. ${ }^{59}$ Crucially, this approach is assumptionfree and allows a reliable comparison of dispersity between different populations via a user-friendly macro. While this approach was first presented on colloidal populations, it can be equally applied to pore size or molecular weight distributions. To this end, the absolute entropy $(E)$ results obtained herein are shown in Table S2. $\dagger$ In the case of the BCP molecular weight, the absolute entropy value reduced from 8150 (non-fractionated BCP) to 6090 (A17), 3760 (A18) and 5490 (A19) after the fractionation. A similar tendency was found for the pore size distribution. EP analysis provided an entropy value $E$ for the non-fractionated BCP of 28.1, which decreased as a consequence of fractionation to 14.0 (A19), 16.5 (A18) and 23.1 (A17), respectively. In comparison, AFM image analysis led to entropy values of 8.16 (as-synth), 5.00 (A19), 5.40 (A18) and 5.42 (A17), respectively. Linear regression of both variables (Fig. 5C) confirms the direct relationship between the dispersity of the BCP structure-directing agent $M_{\mathrm{w}}$ and the dispersity in pore size of the final inorganic porous structure. This correlation was found to be more pronounced in the case of the EP measurements $(p$ value $=0.049)$ than in the case of the AFM data $(p$-value $=0.205)$. While the different techniques are consistent in their findings, some deviation may be related to the fact that AFM refers to the topology of a limited number of pores on the sample surface, while EP determines pore size by capillary condensation occurring in the bulk of the film. Moreover AFM lateral resolution can be affected by tip convolution effects, and therefore some discrepancies between the data obtained by the different techniques may be expected. ${ }^{50}$ Nevertheless, a narrower $M_{\mathrm{w}}$ distribution of the BCP induced in all cases a lower pore size dispersity in the final mesoporous film. This provides further evidence that the herein presented approach of BCP chromatographic fractionation not only offers a straightforward method to obtain an extensive BCP library with little synthetic effort but also reduced dispersity in inorganic mesoporous films.

We note that out approach is fundamentally different to the more common route of pore expansion by supramolecular coassembly with swelling agents that selectively interact with the pore-forming block, e.g. benzene derivatives, ${ }^{60,61}$ homopolymers, ${ }^{55,62}$ carboxylic acids, ${ }^{63,64}$ or solvents such as toluene or xylene. ${ }^{65,66}$ In contrast to the herein presented strategy, swelling agents present multiple challenges, i.e. macroscopic phase separation, increase in the pore size dispersity, multimodal pore size distribution and a decrease in the longrange order of the structure, ${ }^{67}$ which limits their applicability in particular when both mean pore diameter and dispersity are important. Our findings are equally relevant for other BCP techniques, such as sequential infiltration synthesis ${ }^{68,69}$ or aqueous metal reduction, ${ }^{70,71}$ where nanostructured BCP films serve as scaffolds for multistep fabrication procedures post self-assembly.

\section{Conclusions}

Herein, we establish a route to pore size control in mesoporous inorganic thin film architectures based on fractionation by size exclusion chromatography of a polydisperse BCP. The various $M_{\mathrm{w}}$ BCP fractions served for fabrication of aluminosilicate mesoporous architectures via co-assembly. The combination of characterization by AFM, GISAXS and EP allowed the establishment of a close relationship between the molecular properties of the structure-directing BCP and the resulting mesoporous network, with significant variations in the centerto-center distance and pore sizes. Importantly, a close relationship between the entropic dispersity calculations of the BCP and the formed mesopores underlines the importance of polymer dispersity in the initial feedstock and highlights the relevance of the herein presented approach.

\section{Conflicts of interest}

There are no conflicts to declare.

\section{Acknowledgements}

AAF, MJF and SG acknowledge funding by EPSRC New Investigator Award (EP/R035105/1). BR is grateful for an EPSRC Industrial Case Award (EP/M506448/1) in support of BASF. JS acknowledges the EPSRC (EP/L01646X), the MRC (MR/ R000328/1), University College London and University of Nottingham CDT in Advanced Therapeutics and Nanomedicines for financial support. LY is grateful for an 
Inspiration Grant by the UCL Centre for Nature Inspired Engineering (EPSRC Award, EP/K038656/1). The authors thank Dr Han $\mathrm{Wu}$ and the EPSRC CNIE research facility service (EPSRC Award, EP/K038656/1) at University College London for the collection of GISAXS data and Dr Alex Obiakor from Tosoh Bioscience for technical advice on column fractionation.

\section{Notes and references}

1 C. Roychaudhuri, Sens. Actuators, B, 2015, 210, 310-323.

2 S. Alberti, P. Y. Steinberg, G. Giménez, H. Amenitsch, G. Ybarra, O. Azzaroni, P. C. Angelomé and G. J. A. A. SolerIllia, Langmuir, 2019, 35, 6279-6287.

3 N. Reta, C. P. Saint, A. Michelmore, B. Prieto-Simon and N. H. Voelcker, ACS Appl. Mater. Interfaces, 2018, 10, 60556072.

4 W. Kim, S. Y. Choi, Y. M. Jeon, S. K. Lee and S. H. Kim, ACS Appl. Mater. Interfaces, 2014, 6, 11484-11492.

5 E. Doustkhah, J. Lin, S. Rostamnia, C. Len, R. Luque, X. Luo, Y. Bando, K. C.-W. Wu, J. Kim, Y. Yamauchi and Y. Ide, Chem. - Eur. J., 2019, 25, 1614-1635.

6 J. Shao, S. Yang, L. Lei, Q. Cao, Y. Yu and Y. Liu, Chem. Mater., 2016, 28, 7134-7144.

7 P. Docampo, S. Guldin, T. Leijtens, N. K. Noel, U. Steiner and H. J. Snaith, Adv. Mater., 2014, 26, 4013-4030.

8 B. Reid, A. Taylor, Y. Chen, B. Schmidt-Hansberg and S. Guldin, ACS Appl. Mater. Interfaces, 2018, 10, 1031510321.

9 C. Sanchez, C. Boissière, D. Grosso, C. Laberty and L. Nicole, Chem. Mater., 2008, 20, 682-737.

10 P. Wang, M. Wang, F. Liu, S. Ding, X. Wang, G. Du, J. Liu, P. Apel, P. Kluth, C. Trautmann and Y. Wang, Nat. Commun., 2018, 9, 569.

11 E. A. Jackson and M. A. Hillmyer, ACS Nano, 2010, 4, 35483553.

12 J. F. Angiolini, M. Stortz, P. Y. Steinberg, E. Mocskos, L. Bruno, G. Soler-Illia, P. C. Angelomé, A. Wolosiuk and V. Levi, Phys. Chem. Chem. Phys., 2017, 19, 26540-26544.

13 P. Y. Steinberg, M. M. Zalduendo, G. Giménez, G. J. A. A. Soler-Illia and P. C. Angelomé, Phys. Chem. Chem. Phys., 2019, 21, 10347-10356.

14 H. Y. Lian, Y. H. Liang, Y. Yamauchi and K. C.-W. Wu, J. Phys. Chem. C, 2011, 115, 6581-6590.

15 R. K. Kankala, Y. Han, J. Na, C. Lee, Z. Sun, S. Wang, T. Kimura, Y. S. Ok, Y. Yamauchi, A. Chen and K. C. -W. Wu, Adv. Mater., 2020, 32, 1907035.

16 E. Nagy, Basic Equations of Mass Transport Through a Membrane Layer, Elsevier, 2019.

17 A. Walcarius, Electroanalysis, 2015, 27, 1303-1340.

18 P. C. A. Alberius, K. L. Frindell, R. C. Hayward, E. J. Kramer, G. D. Stucky and B. F. Chmelka, Chem. Mater., 2002, 14, 3284-3294.

19 E. L. Crepaldi, G. J. A. A. Soler-Illia, D. Grosso, F. Cagnol, F. Ribot and C. Sanchez, J. Am. Chem. Soc., 2003, 125, 9770-9786.
20 M. Nedelcu, J. Lee, E. J. W. Crossland, S. C. Warren, M. C. Orilall, S. Guldin, S. Hüttner, C. Ducati, D. Eder, U. Wiesner, U. Steiner and H. J. Snaith, Soft Matter, 2009, 5, 134-139.

21 H. N. Lokupitiya, A. Jones, B. Reid, S. Guldin and M. Stefik, Chem. Mater., 2016, 28, 1653-1667.

22 M. Templin, A. Franck, A. Du Chesne, H. Leist, Y. Zhang, R. Ulrich, V. Schadler and U. Wiesner, Science, 1997, 278, 1795-1798.

23 A. Hozumi, H. Sugimura, K. Hiraku, T. Kameyama and O. Takai, Chem. Mater., 2000, 12, 3842-3847.

24 S. Guldin, P. Kohn, M. Stefik, J. Song, G. Divitini, F. Ecarla, C. Ducati, U. Wiesner and U. Steiner, Nano Lett., 2013, 13, 5329-5335.

25 B. Reid, A. Taylor, A. Alvarez-Fernandez, M. H. Ismael, S. Sharma, B. Schmidt-Hansberg and S. Guldin, ACS Appl. Mater. Interfaces, 2019, 11, 19308-19314.

26 J. Noolandi and K. M. Hong, Macromolecules, 1983, 16, 1443-1448.

27 E. Bloch, P. L. Llewellyn, T. Phan, D. Bertin and V. Hornebecq, Chem. Mater., 2009, 21, 48-55.

28 S. Park, D. Y. Ryu, J. K. Kim, M. Ree and T. Chang, Polymer, 2008, 49, 2170-2175.

29 N. A. Lynd, A. J. Meuler and M. A. Hillmyer, Prog. Polym. Sci., 2008, 33, 875-893.

30 H. Feng, X. Lu, W. Wang, N. G. Kang and J. W. Mays, Polymer, 2017, 9, 494.

31 M. Zhang, S. M. June, T. E. Long and J. Kong, in Reference Module in Materials Science and Materials Engineering, Elsevier, 2016.

32 N. Hadjichristidis, M. Pitsikalis and H. Iatrou, Adv. Polym. Sci., 2005, 189, 1-124.

33 N. Hadjichristidis and A. Hirao, Anionic Polymerization, Springer Japan, Tokyo, 2015.

34 A. Mühlebach, S. G. Gaynor and K. Matyjaszewski, Macromolecules, 1998, 31, 6046-6052.

35 T. P. Lodge, Macromol. Chem. Phys., 2003, 204, 265-273.

36 A. B. Chang and F. S. Bates, Macromolecules, 2020, 53, 2765-2768.

37 M. Schollenberger and W. Radke, J. Chromatogr. A, 2011, 1218, 7828-7831.

38 W. Kim, J. Han, C. Y. Ryu and H. Yang, J. Polym. Sci., Part B: Polym. Phys., 2006, 44, 3612-3620.

39 J. Lee, K. Lee, J. Park, J. K. Kim and T. Chang, Polymer, 2015, 80, 46-51.

40 M. Janco, D. Berek, A. Önen, C.-H. Fischer, Y. Yagci and W. Schnabel, Polym. Bull., 1997, 38, 681-688.

41 S. Park, D. Cho, J. Ryu, K. Kwon, W. Lee and T. Chang, Macromolecules, 2002, 35, 5974-5979.

42 S. Park, K. Kwon, D. Cho, B. Lee, M. Ree and T. Chang, Macromolecules, 2003, 36, 4662-4666.

43 A. C. Makan, P. Sinha, N. Ngaza, W. Van Aswegen and H. Pasch, Anal. Bioanal. Chem., 2013, 405, 9041-9047.

44 T. von Graberg, P. Hartmann, A. Rein, S. Gross, B. Seelandt, C. Röger, R. Zieba, A. Traut, M. Wark, J. Janek and B. M. Smarsly, Sci. Technol. Adv. Mater., 2011, 12, 025005. 
45 C. Boissiere, D. Grosso, S. Lepoutre, L. Nicole, A. B. Bruneau and C. Sanchez, Langmuir, 2005, 21, 12362-12371.

46 W. Li, J. Liu and D. Zhao, Nat. Rev. Mater., 2016, 1, 16023.

47 M. R. Baklanov, K. P. Mogilnikov, V. G. Polovinkin and F. N. Dultsev, J. Vac. Sci. Technol., B: Microelectron. Nanometer Struct., 2000, 18, 1385-1391.

48 E. L. Malins, C. Waterson and C. R. Becer, J. Polym. Sci., Part A: Polym. Chem., 2016, 54, 634-643.

49 S. Mondini, A. M. Ferretti, A. Puglisi and A. Ponti, Nanoscale, 2012, 4, 5356.

50 A. Alvarez-Fernandez, B. Reid, M. J. Fornerod, A. Taylor, G. Divitini and S. Guldin, ACS Appl. Mater. Interfaces, 2020, 12, 5195-5208.

51 E. Bloch, P. L. Llewellyn, T. Phan, D. Bertin and V. Hornebecq, Chem. Mater., 2009, 21, 48-55.

52 C. Svaneborg and J. S. Pedersen, Macromolecules, 2002, 35, 1028-1037.

53 M. Gordon, Polymer, 1981, 22, 565.

54 J. Billet, S. Vandewalle, M. Meire, N. Blommaerts, P. Lommens, S. W. Verbruggen, K. De Buysser, F. Du Prez and I. Van Driessche, J. Mater. Sci., 2020, 55, 1933-1945.

55 B. Reid, A. Alvarez-Fernandez, B. Schmidt-Hansberg and S. Guldin, Langmuir, 2019, 35, 14074-14082.

56 M. Thommes, Chem. Ing. Tech., 2010, 82, 1059-1073.

57 S. Harrisson, Polym. Chem., 2018, 9, 1366-1370.

58 P. Innocenzi, S. Costacurta, T. Kidchob, L. Malfatti, P. Falcaro and G. Soler-Illia, in Thin Films, Springer, Dordrecht, 2008, pp. 105-123.
59 N. Mac Fhionnlaoich and S. Guldin, Chem. Mater., 2020, 32, 3701-3706.

$60 \mathrm{~J}$. Yi and M. Kruk, Langmuir, 2015, 31, 7623-7632.

61 J. Fan, C. Yu, J. Lei, Q. Zhang, T. Li, B. Tu, W. Zhou and D. Zhao, J. Am. Chem. Soc., 2005, 127, 10794-10795.

62 A. Sarkar, A. Thyagarajan, A. Cole and M. Stefik, Soft Matter, 2019, 15, 5193-5203.

63 A. Álvarez-Fernández, F. Valdés-Bango, R. LosadaAmbrinos, J. I. Martín, M. Vélez, J. M. Alameda and F. J. García Alonso, Polym. Int., 2018, 67, 393-398.

64 A. Alvarez-Fernandez, F. Valdes-Vango, J. I. Martín, M. Vélez, C. Quirós, D. Hermida-Merino, G. Portale, J. M. Alameda and F. J. García Alonso, Polym. Int., 2019, 68, 1914-1920.

65 Y. Li and M. Kruk, RSC Adv., 2015, 5, 69870-69877.

66 L. Huang and M. Kruk, Chem. Mater., 2015, 27, 679689.

67 Q. L. Wu and S. E. Rankin, J. Phys. Chem. C, 2011, 115, 11925-11933.

68 C. Zhou, T. Segal-Peretz, M. E. Oruc, H. S. Suh, G. Wu and P. F. Nealey, Adv. Funct. Mater., 2017, 27, 1701756.

69 Q. Peng, Y. C. Tseng, S. B. Darling and J. W. Elam, ACS Nano, 2011, 5, 4600-4606.

70 A. Alvarez-Fernandez, K. Aissou, G. Pécastaings, G. Hadziioannou, G. Fleury and V. Ponsinet, Nanoscale Adv. , 2019, 1, 849-857.

71 J. Chai, D. Wang, X. Fan and J. M. Buriak, Nat. Nanotechnol., 2007, 2, 500-506. 